\title{
CUBIERTAS DE MACROTÚNELES Y SU EFECTO EN LAS PROPIEDADES NUTRACEÚTICAS DEL CHILE DE AGUA
}

\author{
MACRO TUNNELS COVERINGS AND THEIR EFFECT ON THE \\ NUTRACEUTICAL PROPERTIES OF "CHILE DE AGUA"
}

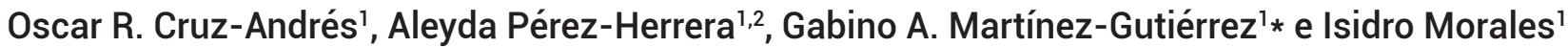

\begin{abstract}
'Instituto Politécnico Nacional, Centro Interdisciplinario de Investigación para el Desarrollo Integral Regional-Unidad Oaxaca, Santa Cruz Xoxocotlán, Oaxaca, México. ${ }^{2}$ Consejo Nacional de Ciencia y Tecnología. Ciudad de México, México.
\end{abstract}

*Autor para correspondencia (gamartinezg@ipn.mx)

\section{RESUMEN}

El chile de agua es una hortaliza endémica de los Valles Centrales de Oaxaca, que a pesar de su importancia económica y para la nutrición humana, su contenido de compuestos bioactivos está poco estudiado. El objetivo fue determinar el contenido de compuestos bioactivos en el fruto del chile de agua (Capsicum annuum L.), cultivado en macrotúneles con diferentes materiales de cubierta. Plántulas de chile de agua del genotipo "Ejutla" fueron cultivadas con acolchado plástico y fertirriego, en macrotúneles con cubiertas de plástico transparente, plástico verde y malla blanca. Cada cubierta correspondió a un tratamiento y el cultivo a cielo abierto fue el testigo. Se utilizó un diseño experimental completamente al azar con tres repeticiones. Las variables respuesta fueron a) climáticas: temperatura, humedad relativa y radiación fotosintéticamente activa integrada (RFAl); b) compuestos bioactivos: fenoles totales, flavonoides y capacidad antioxidante. La temperatura diurna, nocturna y humedad relativa bajo plástico verde aumentaron 28.0, 6.7 y 0.5 $\%$ con respecto a campo abierto. La RFAl en campo abierto superó en $28.3 \%$ a los materiales de cubierta. El plástico verde incrementó significativamente la concentración de fenoles totales y capacidad antioxidante, superando al testigo en 489.1 y $39.0 \%$, respectivamente. La concentración de flavonoides totales de los frutos cultivados en campo abierto fue mayor en al menos 47 $\%$ a los materiales de cubierta evaluados. La concentración de fenoles de los frutos de chile de agua y la capacidad antioxidante se incrementan cuando se cultivan en macrotúneles con cubierta de plástico verde.

Palabras clave: Capsicum annuum, compuestos bioactivos, fenoles, flavonoides, antioxidantes.

\section{SUMMARY}

The "Chile de agua" is an endemic vegetable of the central valleys of Oaxaca, but despite its importance in the economic and human nutrition field, its bioactive compounds has been poorly studied. The objective of this research was to determine the content of bioactive compounds into the fruit of the "Chile de agua" (Capsicum annuum L.), grown under macro tunnels with different cover materials. Seedlings of the "Ejutla" genotype were planted in soil with plastic mulch and fertigation under macro tunnels using transparent and green plastic covers, and white mesh. Each cover corresponded to a treatment, and the open-air cultivation was the control. The response variables were grouped into a) climatic: temperature, relative humidity and Integrated Photosynthetically Active Radiation (IPAR); b) bioactive compounds: total phenols, flavonoids and antioxidant capacity. The daytime and nighttime temperatures and relative humidity under green plastic cover increased $28.0,6.7$ and $0.5 \%$ with respect to the control. The IPAR in open-air cultivation exceeded the cover materials by $28.3 \%$. The green plastic significantly increased the concentration of total phenols and antioxidant capacity by 489.1 and $39.0 \%$ with respect to the control. The concentration of total flavonoids of the fruits grown in open-air cultivation was greater than at least $47 \%$ to the cover materials evaluated. The phenols concentration of "Chile de agua" fruits and their antioxidant capacity increased when they are grown in macro tunnels with green plastic covers.

Index words: Capsicum annuum, bioactive compounds, phenols, flavonoids, antioxidants.

\section{INTRODUCCIÓN}

El chile de agua (Capsicum annuum L.) es una hortaliza endémica de los Valles Centrales de Oaxaca, de importancia económica, social y cultural (Montaño-Lugo et al., 2014), muy apreciado por los habitantes de esta región quienes lo consumen todo el año. Los chiles contienen una amplia variedad de compuestos bioactivos como el ácido ascórbico, vitaminas $\mathrm{C}, \mathrm{A}$ y $\mathrm{E}$, carotenoides, capsaicinoides y compuestos fenólicos (Bae et al., 2014). Los compuestos fenólicos poseen propiedades antioxidantes que pueden ayudar a reducir los daños causados por los procesos de oxidación de los radicales libres en el organismo y las enfermedades asociadas a estos daños (HervertHernández et al., 2010). En las plantas, los fenoles tienen funciones como pigmentación, crecimiento y adaptación a condiciones de estrés (Tucuch-Haas et al., 2017). Estos compuestos y sus proporciones en los frutos de Capsicum spp. pueden variar debido a distintos factores, entre los más importantes son: la variedad o genotipo, la temporada y las condiciones ambientales en que se cultivan y las prácticas agronómicas (Deepa et al., 2007). El manejo de la luz solar en las plantas puede aumentar el rendimiento en biomasa y componentes químicos (Nishimura et al., 2007). La fotosíntesis en la mayoría de las plantas $C_{3}$ se satura a los $500 \mu \mathrm{mol} \cdot \mathrm{m}^{-2} \cdot \mathrm{s}^{-1}$ y tanto mallas como plásticos reducen la intensidad de la radiación solar, al mismo 
tiempo que modifican la temperatura y humedad relativa, lo cual afecta a la traspiración, fotosíntesis, respiración y otros procesos (Chang et al., 2008). Rivera-Pastrana et al. (2007) reportaron que los rayos ultravioleta modifican las propiedades nutricionales de frutas y hortalizas al favorecer la acumulación de fitoalexinas y el incremento de algunas vitaminas y antioxidantes. No existe suficiente información sobre el contenido nutricional de chile de agua bajo estructuras de protección, por lo que el objetivo del presente estudio fue evaluar el contenido de algunas propiedades nutraceúticas de frutos de chile de agua producidos en macrotúneles con diferentes materiales de cubierta y a campo abierto.

\section{MATERIALES Y MÉTODOS}

El experimento se realizó de junio a agosto del 2016 en el campo experimental y en el laboratorio de nutrigenómica del CIIDIR IPN Unidad Oaxaca, en Santa Cruz Xoxocotlán, Oaxaca, México (170 01' 30.3" N y $96^{\circ} 43^{\prime} 12.5^{\prime \prime}$ O, a 1530 metros sobre el nivel del mar). Se construyeron nueve macrotúneles de $4 \times 15 \times 2$ m de ancho, largo y alto respectivamente, cubiertos con malla blanca, plástico transparente y plástico verde. Cada material de cubierta fue un tratamiento y el testigo fue el cultivo a campo abierto. Se utilizaron semillas nativas de Ejutla de Crespo, Oaxaca. A los 45 días después de su germinación, las plántulas se trasplantaron en suelo con acolchado plástico, a una densidad de tres plantas $\mathrm{m}^{-2}$. Las características del suelo fueron: textura arenosa con $91 \%$ de arena, $2.7 \%$ de limo y $6.3 \%$ de arcilla, densidad aparente de $1.55 \mathrm{~g} \mathrm{~cm}^{-3}$, capacidad de campo 8.5 \% y punto de marchitez $3.5 \%$, infiltración básica de $6.3 \mathrm{~cm}$ $\mathrm{h}^{-1}$, materia orgánica $1.6 \%$, CE del extracto de saturación de $1.2 \mathrm{dS} \mathrm{m}^{-1}$ y 92, 142, 4318, 350 y $253 \mathrm{mg} \mathrm{kg}^{-1} \mathrm{de} \mathrm{P,} \mathrm{K,} \mathrm{Ca,}$ Mg y SO 4 extractables, respectivamente, más $4 \mathrm{mg} \mathrm{kg}^{-1}$ de $\mathrm{N}-\mathrm{NO}_{3}$ (Norma Oficial Mexicana NOM-021_RECNAT-200, SEMARNAT, 2001). La prevención y control de plagas y enfermedades se realizó con productos biológicos y el control de malezas fue manual.

El diseño experimental utilizado fue completamente al azar con tres repeticiones y cinco plantas como unidad experimental. La fertirrigación fue de acuerdo a Segura y Cadahía (2000) y se aplicó con cintilla de riego por goteo. La temperatura diurna, nocturna y humedad relativa del ambiente se registró en todo el ciclo de cultivo cada 5 minutos las 24 horas del día, con sensores $\mathrm{HOBO}{ }^{\circledR}$ Pro V2 (Onset, EUA). La Radiación Fotosintéticamente Activa (RFA) se obtuvo con un sensor lineal cuántico MQ300 (Apogee Instruments Inc., EUA) y se transformó a Radiación Fotosintéticamente Activa Integrada (RFAl) expresada en $\mathrm{mol} \mathrm{m}^{-2} \mathrm{~d}^{-1}$ (Chang et al., 2008). Se realizaron cuatro cortes en total, seleccionando una muestra aleatoria de 20 frutos por repetición, a los cuales se les separó el pericarpio y se llevó a peso constante en un horno de convección por gravedad (Modelo H-33, Industrias BG, México). La cuantificación de los fenoles totales se hizo por el método Folin-Ciocalteu (Singleton y Rossi, 1965) y se expresó en mg EAG $\mathrm{g}^{-1}$ de materia seca. El contenido de flavonoides totales se obtuvo de acuerdo a Chen et al. (2014) y se expresó como mg QE g ${ }^{-1}$ de materia seca. La capacidad antioxidante se determinó por la técnica descrita por Matthaus (2002).

Los datos obtenidos fueron sometidos a un análisis de varianza y a comparación de medias (Tukey, $\mathrm{P} \leq 0.05$ ). La relación entre las concentraciones de fenoles, flavonoides y la capacidad antioxidante de los frutos se determinó con el coeficiente de correlación lineal de Pearson. Los análisis se realizaron con el programa Minitab® 18.1 (Minitab, Inc., Pennsylvania, USA).

\section{RESULTADOS Y DISCUSIÓN}

El análisis de varianza mostró diferencias estadísticas significativas en la mayoría de las variables evaluadas, excepto en la humedad relativa (Cuadro 1).

En el interior de los macrotúneles se encontraron diferencias significativas $(P \leq 0.05)$ en la temperatura ambiental diurna y nocturna, las cuales fueron mayores que en campo abierto (Cuadro 2). La temperatura diurna y nocturna bajo plástico verde aumentaron 28.0 y $6.7 \%$ con respecto a campo abierto. Bajo las cubiertas y campo abierto no se encontraron diferencias significativas en la humedad relativa, con un valor promedio de $68.3 \%$. La mayor RFAl se obtuvo en campo abierto (54.8 $\left.\mathrm{mol} \mathrm{m}^{-2} \mathrm{dia}^{-1}\right)$ (Cuadro 2).

El contenido de flavonoides fue mayor en los frutos producidos en campo abierto (Figura 1a), comparado con los obtenidos bajo las cubiertas, lo cual puede ser el resultado de una exposición significativamente mayor a la luz solar, ya que como lo indican Harborne y Williams (2000), el incremento en la síntesis de flavonoides (flavonas y flavonoles) es un mecanismo para la protección de los tejidos epidérmicos contra la radiación UV-B. De esta manera, los compuestos fotoestabilizantes adicionados en los plásticos de cubierta pudieron bloquear parte de dicha radiación (Castilla, 2004) y causar una menor síntesis de flavonoides.

Bajo la cubierta de plástico verde el contenido de fenoles totales en los frutos incrementó 14.48 y 2.96 mg EAG $\mathrm{g}^{-1}$ de materia seca, respectivamente (Figura 1a), lo que representa $489.1 \%$ al obtenido en campo abierto. Santos et al. (2014) indicaron que la exposición de las plantas a la luz y a la temperatura influye en la síntesis de fenoles y flavonoides, contribuyendo ampliamente a su potencial antioxidante, mostrando una relación entre compuestos 
Cuadro 1. Cuadrados medios y significancia del análisis de varianza de los parámetros ambientales y nutraceúticos del chile de agua bajo diferentes cubiertas de macrotúneles y campo abierto.

\begin{tabular}{|c|c|c|c|c|c|c|c|c|}
\hline \multirow{3}{*}{$\begin{array}{l}\text { Fuente de } \\
\text { variación }\end{array}$} & \multirow{3}{*}{$\mathrm{GL}$} & \multicolumn{7}{|c|}{ Cuadrados medios } \\
\hline & & \multicolumn{4}{|c|}{ Ambientales } & \multicolumn{3}{|c|}{ Nutraceúticos } \\
\hline & & TD & TN & $\mathrm{HR}$ & RFAl & FEN & FLA & CA \\
\hline Cubiertas & 3 & $23.99 *$ & $4.97 *$ & $127.83 \mathrm{~ns}$ & $179.13 *$ & $102.74 *$ & $36.86 *$ & $0.04 *$ \\
\hline Error & 8 & 0.16 & 0.18 & 56.57 & 4.75 & 0.39 & 1.17 & 0.001 \\
\hline
\end{tabular}

GL: Grados de libertad; TD: Temperatura diurna; TN: Temperatura nocturna; HR: Humedad relativa; RFAl: Radiación Fotosintéticamente Activa Integrada; FEN: Fenoles totales; FLA: Flavonoides totales; CA: Capacidad antioxidante; * y ns: Significativo $(P \leq 0.05)$ y no significativo, respectivamente.

Cuadro 2. Parámetros ambientales en el interior de los macrotúneles con diferentes materiales de cubiertas y campo abierto.

\begin{tabular}{lcccc}
\hline Parámetro & CA & M & PT & PV \\
\hline Temperatura diurna $\left({ }^{\circ} \mathrm{C}\right)$ & $22.0 \pm 0.3 \mathrm{c}+$ & $25.5 \pm 0.3 \mathrm{~b}$ & $27.6 \pm 0.2 \mathrm{a}$ & $28.3 \pm 0.6 \mathrm{a}$ \\
Temperatura nocturna $\left({ }^{\circ} \mathrm{C}\right)$ & $17.7 \pm 0.4 \mathrm{c}$ & $18.3 \pm 0.4 \mathrm{~b}$ & $20.5 \pm 0.4 \mathrm{a}$ & $19.8 \pm 0.5 \mathrm{a}$ \\
Humedad relativa $(\%)$ & $73.8 \pm 0.6 \mathrm{a}$ & $62.6 \pm 0.3 \mathrm{a}$ & $62.8 \pm 2.8 \mathrm{a}$ & $74.2 \pm 2.8 \mathrm{a}$ \\
RFAl $\left(\mathrm{mol} \mathrm{m}^{-2} \mathrm{dia}^{-1}\right)$ & $54.8 \pm 1.7 \mathrm{a}$ & $39.3 \pm 1.8 \mathrm{~b}$ & $39.0 \pm 2.2 \mathrm{~b}$ & $39.6 \pm 2.9 \mathrm{~b}$ \\
\hline
\end{tabular}

CA: Campo abierto; M: Malla blanca, PT: Plástico transparente; PV: Plástico verde; RFAl: Radiación Fotosintéticamente Activa Integrada. \pm Desviación estándar; † Medias con la misma letra por fila son estadísticamente iguales (Tukey, 0.05).
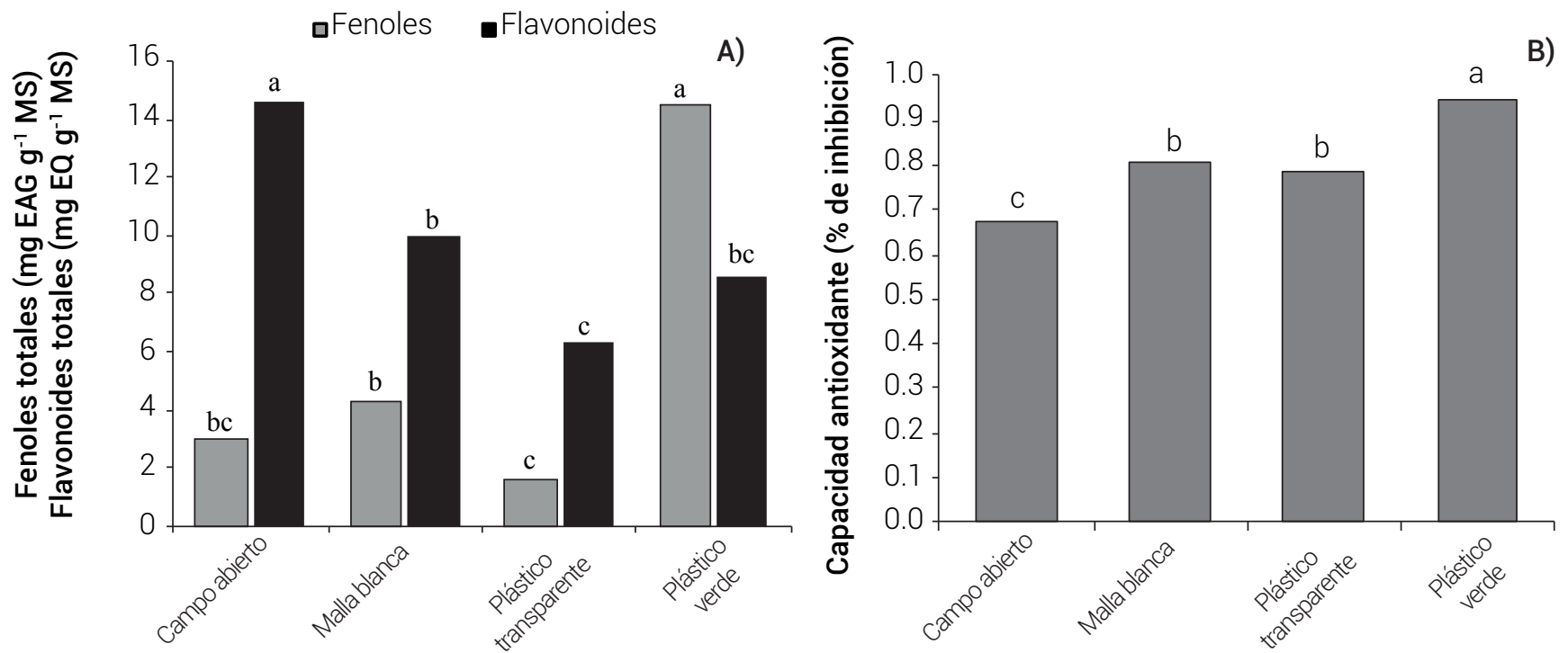

Figura 1. Concentración de: A) fenoles y flavonoides totales y B) capacidad antioxidante de frutos de chile de agua cultivados en macrotúneles con diferentes cubiertas y a campo abierto. Barras con la misma letra en cada color son estadísticamente iguales (Tukey, 0.05).

fenólicos y la eliminación de radicales libres (Zovko et al., 2010). También, Mendoza-Sánchez et al. (2015) encontraron que un contenido fenólico de 5.04 mg de EAG por gramo de materia seca aumentó el potencial antioxidante de los frutos del chile jalapeño. La capacidad antioxidante de los frutos cultivados bajo el plástico verde fue 20, 17 y $39 \%$ mayor al obtenido en el plástico trasparente, malla y campo abierto, respectivamente (Figura 1b), lo cual muestra una correlación lineal positiva con la concentración de fenoles $(r=0.833$; $P<0.01)$, pero una correlación negativa con la concentración de flavonoides $(r=-0.617 ; P<0.01)$.

\section{CONCLUSIONES}

Los diferentes materiales de cubierta modificaron el microclima en el interior de los macrotúneles, excepto la 
humedad relativa. La RFAl disminuyó en 28.3 \% en el interior de los macrotúneles con respecto a campo abierto. La concentración de fenoles totales de los frutos de chile de agua y el aumento de la capacidad antioxidante se incrementó cuando fueron cultivados en macrotúneles con cubierta de plástico verde. La mayor concentración de flavonoides se encontró en los chiles cultivados a campo abierto. El contenido de fenoles totales mostró correlación positiva con la capacidad antioxidante y correlación negativa con el contenido de flavonoides.

\section{AGRADECIMIENTOS}

Los autores agradecen al Instituto Politécnico Nacional, por el financiamiento otorgado al proyecto SIP- 20160437 y al CONACYT, por el Proyecto Cátedras 2014 y Beca 423967 para estudios Doctorales.

\section{BIBLIOGRAFÍA}

Bae H., G.K. Jayaprakasha, K. Crosby, K. S. Yoo, D. I. Leskovar, J. Jifon and B. S. Patil (2014) Ascorbic acid, capsaicinoid, and flavonoid aglycone concentrations as a function of fruit maturity stage in greenhouse-grown peppers. Journal of Food Composition and Analysis 33:192-202

Castilla P. N. (2004) Invernaderos de plástico: Tecnología y Manejo. Ediciones Mundi-Prensa, España, 462 pp.

Chang X., P. G. Alderson and C. J. Wright (2008) Solar irradiance level alters the growth of basil (Ocimum basilicum L.) and its content of volatile oils. Environmental Experiment Botany 63:216-223.

Chen L., X. Xin, Q. Yuan, D. Su and W. Liu (2014) Phytochemical properties and antioxidant capacities of various colored berries. Journal of the Science of Food and Agriculture 94(2):180-188.

Deepa N., C. Kaur, B. George, B. Singh and H. C. Kapoor (2007) Antioxidant constituents in some sweet pepper (Capsicum annuum L.) genotypes during maturity. LWT-Food Science and Technology 40:121-129

Harborne J. B. and C. A. Williams (2000) Advances in flavonoid research since 1992. Phytochemistry 55:481-504.
Hervert-Hernández D., S. G. Sáyago and I. Goñi (2010) Bioactive compounds of four hot pepper varieties (Capsicum annuum L.), antioxidant capacity, and intestinal bioaccessibility. Journal of Agricultural and Food Chemistry 58:3399-3406.

Matthaus B. (2002) Antioxidant activity of extracts obtained from residues of different oilseeds. Journal of Agricultural and Food Chemistry 50:3444-3452.

Mendoza-Sánchez L. G., M. R. Mendoza-López, O. García-Barradas, E. Azuara-Nieto, L. A. Pascual-Pineda y M. Jiménez-Fernández (2015) Propiedades fisicoquímicas y antioxidantes del chile jalapeño (Capsicum annuum var. annuum) durante el almacenamiento. Revista Chapingo, Serie Horticultura 3:229-241.

Montaño-Lugo M. L., V. A. Velasco V., J. Ruíz L., G. V. Campos Á., G. Rodríguez 0. y L. Martínez M. (2014) Contribución al conocimiento etnobotánico del chile de agua (Capsicum annuum L.) en los Valles Centrales de Oaxaca, México. Revista Mexicana de Ciencias Agrícolas 5:503-511.

Nishimura T., S. M. Zobayed, T. Kozai and E. Goto (2007) Medicinally important secondary metabolites and growth of Hypericum perforatum L. plants as affected by light quality and intensity. Environmental Control in Biology 45:113-120.

Rivera-Pastrana D. M., A. A. Gardea-Béjar, M. A. Martínez-Téllez, M. RiveraDomínguez y G. A. González-Aguilar (2007) Efectos bioquímicos postcosecha de la irradiación UV-C en frutas y hortalizas. Revista Fitotecnia Mexicana 30(4):361-372.

Santos J., M. Oliveira, E. Ibáñez and M. Herrero (2014) Phenolic profile evolution of different ready-to-eat baby leaf vegetables during storage. Journal of Chromatography 1327:118-131.

Segura M. L. \& Cadahía, C. (2000) Fertirrigación de cultivos hortícolas. In: Fertirrigación, Cultivos Hortícolas y Ornamentales. C. Cadahía (ed.). Mundi-Prensa, España, pp: 343-415.

SEMARNAT, Secretaría de Medio Ambiente y Recursos Naturales (2001) Norma Oficial Mexicana NOM-021-RECNAT-20bae00. Diario Oficial de la Federación (DOF).

Singleton V. L. and J. A. Rossi (1965) Colorimetry of total phenolics with phosphomolybdic-phosphotungstic acid reagents. American Journal of Enology and Viticulture 16:144-158.

Tucuch-Haas C., G. Alcántara-González, Y. Salinas-Moreno, L. I. Trejo-Téllez, V. H. Volke-Haller y A. Larqué-Saavedra (2017) Aspersión foliar de ácido salicílico incrementa la concentración de fenoles en el grano de maíz. Revista Fitotecnia Mexicana 40(2):235-238.

Zovko K. M., D. Kremer, K. Karlovic and I. Kosalec (2010) Evaluation of antioxidant activities and phenolic content of Berberis vulgaris $\mathrm{L}$. and Berberis croatica Horvat. Food and Chemical Toxicology 48(8-9):2176-2180. 